Article

\title{
Collision Avoidance Method Using Vector-Based Mobility Model in TDMA-Based Vehicular Ad Hoc Networks
}

\author{
Jung-Hyun Bang and Jung-Ryun Lee * $\mathbb{D}$ \\ School of Electrical and Electronics Engineering, Chung-Ang University, 84 Heukseok-ro, Dongjak-gu, \\ Seoul 06974, Korea; bjh929@cau.ac.kr \\ * Correspondence: jrlee@cau.ac.kr; Tel.: +82-2-820-5820
}

Received: 12 May 2020; Accepted: 16 June 2020; Published: 18 June 2020

\begin{abstract}
The rapid development of wireless technology has accelerated the development of Vehicular Ad Hoc Networks (VANETs) to support accident prevention and the safety of a vehicle driver. VANET is a form of Mobile Ad Hoc Network (MANET), and it differs from MANET in that the network topology of a VANET changes dynamically in response to the high mobility of a vehicle and the unstable link quality due to various types of road patterns. Since most access and merging conflicts occur due to vehicle movement patterns and traffic conditions, the collision rate can be reduced if each vehicle can predict the location, movement direction, and resource occupancy information of other vehicles. In this paper, we propose a collision avoidance method based on the vehicle mobility prediction model in TDMA-based VANET. The proposed algorithm allocates time-slots of TDMA to avoid access and merging collisions by predicting the mobility of nearby vehicles using control time-slot occupancy information, vehicle ID, hop information, vehicle movement direction, and longitude and latitude of a vehicle. Simulation results show that the proposed algorithm can reduce access and merging collision rates compared with other legacy algorithms, and the performance gain of the proposed algorithm is enhanced in road environments when traffic density is high and where vehicles have high mobility and change their travel directions frequently.
\end{abstract}

Keywords: VANET; merging collision; access collision; mobility model

\section{Introduction}

Recently, the development of Vehicular Ad Hoc Networks (VANETs) has been accelerated in order to support accident prevention and the safety of vehicle drivers. The number of vehicles continues to increase worldwide, and millions of people are killed or injured by fatal accidents every year [1]. In spite of speed regulations and safety sign boards installed around the roads, technological developments in intelligent vehicle communication networks are essential for accident prevention and the safety of drivers. VANET is a form of Mobile Ad Hoc Network (MANET), but it differs from MANET in that the network topology of VANET dynamically changes, link quality is usually unstable due to the high mobility of a vehicle, there are various types of road patterns, sufficient energy and storage are supported for mobile devices installed in a vehicle, and various quality of service (QoS) are required for VANET services [2]. The applications for VANET can be divided into the following three services: safety services, traffic management, and user-oriented services. Safety services require special requirements of QoS, such as bounded transmission delays and low access delays, both of which are mandatory to offer the highest level of user safety. On the other hand, traffic management services are required to be provided according to vehicle density and data traffic load, and user-oriented services need a high bandwidth. As such, from the perspective of the various requirements of VANET services, 
medium access control (MAC) plays an important role in VANET. In particular, it is noticed that, from the perspective of safety services, MAC should be able to periodically broadcast a safety-related message that includes useful information such as vehicle accident alerts and road conditions [3].

Wireless Access in Vehicular Environment (WAVE) based on IEEE 802.11p and IEEE 1609 standards is a representative standardization study of Intelligent Transport Systems (ITSs) using VANET technologies, which is designed to be suited for the characteristics of vehicles such as high mobility and short communications time. WAVE is based on competition-based resource allocation that provides priority-based access using both EDCA (Enhanced Distributed Channel Access) and Carrier Sense Multiple Access with Collision Avoidance (CSMA/CA). The contention-based resource allocation scheme can guarantee low data transmission delay and high user QoS when traffic density is low. However, if offered traffic load and the mobility of the terminal is high, collision probability increases. Accordingly, the data transmission delay increases and the user QoS deteriorates, so that it is not possible to effectively prevent the accident in the VANET environment.

To overcome the shortcomings of IEEE 802.11p and facilitate efficient communication in VANET, Time Division Multiple Access (TDMA)-based contention-free MAC protocols have been proposed. There are two types of TDMA-based MAC protocols for VANET systems; distributed TDMA and centralized TDMA. In distributed TDMA, each vehicle allocates and manages its own resources and communication with 1-hop neighbors [4]. In contrast, in centralized TDMA, the resource allocation/management of each vehicle is managed by a roadside unit (RSU) or a central coordinator $[5,6]$. However, vehicles traveling at high speed have short dwelling times to stay within the coverage of an RSU, which results in frequent resource collisions. In addition, to support seamless communication for fast moving vehicles, the number of RSUs installed per unit-area increases, and consequently, there are disadvantages of mutual interference between RSUs and collision of resource allocation by hidden nodes in the cluster.

In a TDMA-based VANET system, a frame is usually divided into a control channel and a service channel. Each vehicle generates and transmits a safety message including vehicle information, and this message is periodically delivered to 1-hop neighbor vehicles via the control channel. The service channel is used to transmit non-secure messages such as video, audio, and emergency messages. A resource collision in the control channel affects the QoS of the application provided by the service channel of each vehicle. Specifically, in a TDMA-based VANET protocol, there are two kinds of collisions. First, an access collision occurs when multiple vehicles within a 2-hop communication range attempt to acquire the same available time-slot at the same time, as shown in Figure 1. Second, a merging collision occurs when multiple vehicles using the same time-slot get close to each other within a 2-hop communication range due to the mobility of a vehicle. For example, as in Figure 2, suppose that vehicle 3 and vehicle 4 occupy the second time-slot of the frame. At first, since the two vehicles are more than two hops away from each other, there is no resource conflict. However, the two vehicles are moving towards each other, which will result in a merging collision in the near future.

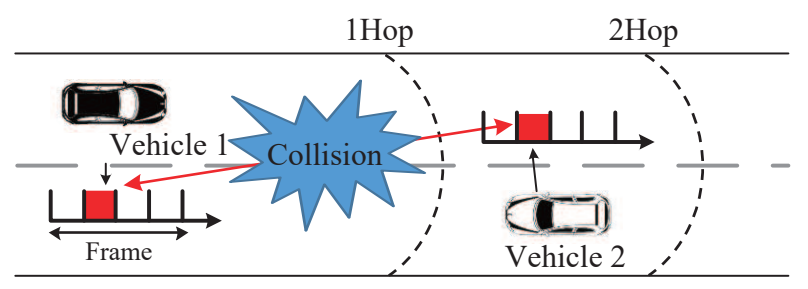

Figure 1. Access collision. 


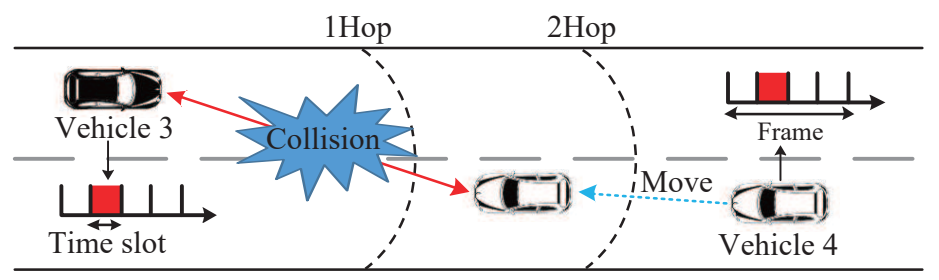

Figure 2. Merging collision.

In this paper, we propose a prediction-based collision avoidance algorithm in VANET. The proposed algorithm is designed to recognize the existence of vehicles far away more than 2 hops and the collision probability of vehicles more than 2 hops away are effectively predicted in the proposed algorithm by using the vector-based prediction model. Because the proposed algorithm is based on the vector mobility of a vehicle, it has the advantage of predicting vehicle movements against other protocols, which enables the proposed algorithm to enhance the performance of collision rate compared to other direction-based algorithms.

The remainder of this paper is organized as follows. Section 2 explains related works on representative distributed TDMA-based MAC protocols VANET. Section 3 explains the operational procedure, mobility model, and frame/message structures of the proposed model. Section 4 provides the analysis of access/merging collision probabilities under the proposed model. In Section 5, we evaluate the performance of the proposed algorithm and compare its performance with those of other collision avoidance methods. We conclude in Section 6.

\section{Related Works}

In a TDMA-based VANET protocol, each vehicle can occupy more than one time-slot per frame; as such, it provides the following advantages over the competition-based IEEE 802.11p standard [3].

- Equal access to the channel for all vehicle nodes.

- Efficient channel utilization without collisions.

- High reliability of communications.

- Deterministic access time even for high traffic loads.

- $\quad$ QoS for real-time applications.

In VANET environments, the network topology changes frequently because vehicles are moving at various speeds. Therefore, a variety of distributed TDMA-based MAC protocols have been proposed considering the advantages of TDMA and the characteristics of VANET. AD HOC Medium Access Control (ADHOC MAC) is a basic distributed TDMA-based MAC protocol designed to provide efficient and reliable data transmission in VANET environments [7]. As shown in Figure 3, each vehicle periodically generates and broadcasts an FI (frame information) message including information about its own 1-hop neighbors and its own time-slot allocation. Because a vehicle receiving the FI message recognizes the neighbors of the vehicle broadcasting the FI message, all vehicles can obtain information about the existence and resource allocation status of 2-hop neighbor vehicles. A new vehicle entering the network listens to the channels of each frame to obtain the unused time-slot information and selects an available time-slot for transmitting data in the next frame based on this information. If the vehicle successfully secures the time-slot, it will continue to use the same time-slot until a collision occurs or the connection is released. However, ADHOC MAC has disadvantages in that it does not take into consideration the moving direction and speed of the vehicle. 


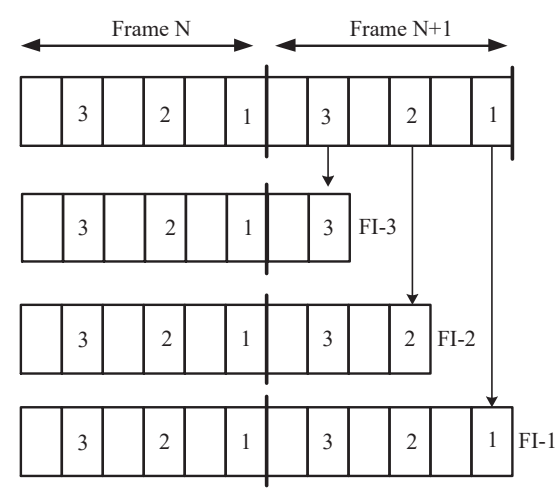

Figure 3. Frame architecture of ADHOC Medium Access Control (MAC).

Vehicular Ad Hoc Network MAC (VeMAC) is a distributed TDMA-based VANET protocol that improves on the disadvantages of ADHOC MAC by considering the moving direction of a vehicle [8]. Each vehicle, like the ADHOC MAC, broadcasts an FI message to its 1-hop neighbor vehicles. All vehicles can obtain information about the presence and resource allocation status of 2-hop neighbor vehicles. In VeMAC, each frame is evenly divided considering the direction of the vehicles. As shown in Figure 4, a frame is divided into two sub-frames. A vehicle traveling to the left occupies the left direction time-slot, and a vehicle traveling to the right occupies the right time-slot. Each vehicle may occupy a time-slot in other directions if there is no remaining time-slot. Because used time-slots are classified according to the moving direction of the vehicle, this results in lowering the merging collision rate. On the other hand, VeMAC did not take into account the relative speed difference between the vehicles; therefore, merging and access collisions may occur when there are many vehicles moving at different speeds.
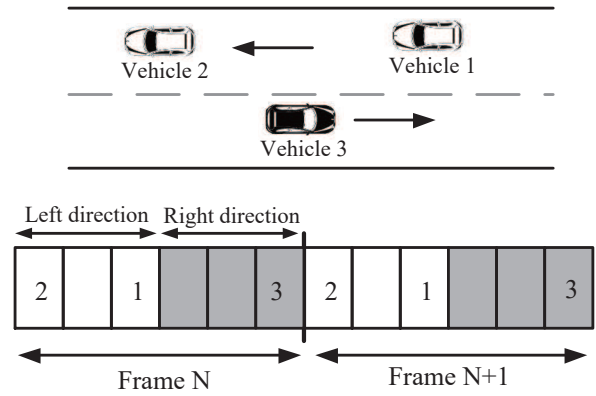

Figure 4. Frame architecture of Vehicular Ad Hoc Network MAC (VeMAC).

The collision-free reservation-based MAC (CFR MAC) is a distributed TDMA protocol that improves on VeMAC by considering the relative speed and traffic flow of each vehicle [9]. Like VeMAC, the resource allocation of each vehicle is divided into two sub-frames and resources are allocated to the vehicles according to their moving directions (left and right). As we just discussed, if vehicles moving in the same direction have different speeds, a merging collision problem may occur. To solve this problem in CFR MAC, the left/right sub-frame of each frame is subdivided into three levels (high, middle, and low), and resources are allocated accordingly, as shown in Figure 5. Each vehicle calculates its relative speed from the difference between its own speed and the average speed of neighbor vehicles. If the relative speed is lower than the standard deviation speed, it is set to have a "low" phase. If it is higher than the standard deviation, it is set to have a "high" phase. The rest of the vehicles are determined to have a "middle" phase. CFR MAC has the advantage of avoiding merging collisions because it allocates resources by considering both the moving direction and speed of each vehicle. However, when traffic density is high in one direction, vehicles may experience many merging collisions due to finely divided resource allocation units. 


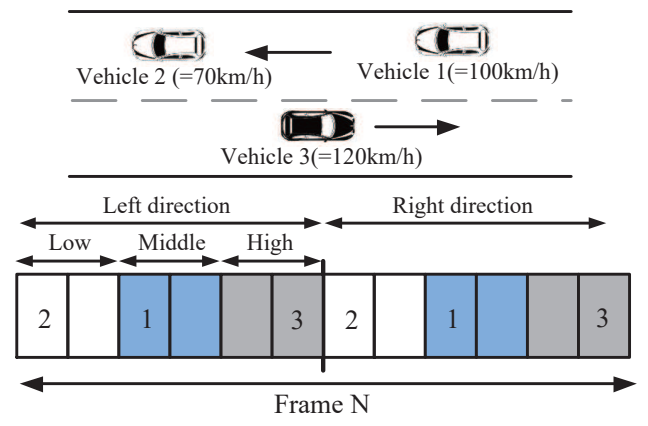

Figure 5. Architecture of collision-free reservation-based MAC (CFR MAC).

Prediction-based TDMA MAC (PTMAC) proposed a method to avoid resource collisions by predicting the position of vehicles using the same resources over a 2-hop communication range [10]. Like ADHOC MAC, PTMAC allocates available time-slots randomly regardless of the vehicle movement direction and speed. Each vehicle stores its occupied time-slot, its own position, its speed, and its moving direction in its FI message and broadcasts it to its own 1-hop neighbor vehicles. As shown in Figure 6, each vehicle can recognize the existence of 2-hop neighbor vehicle(s) using the same time-slot from the FI message and can predict whether there will be resource collision in the near future by estimating the position of vehicles sharing the same time-slot. If a resource collision is predicted, the vehicle abandons the resources occupied by the current frame and selects another unused time-slot in the next frame.

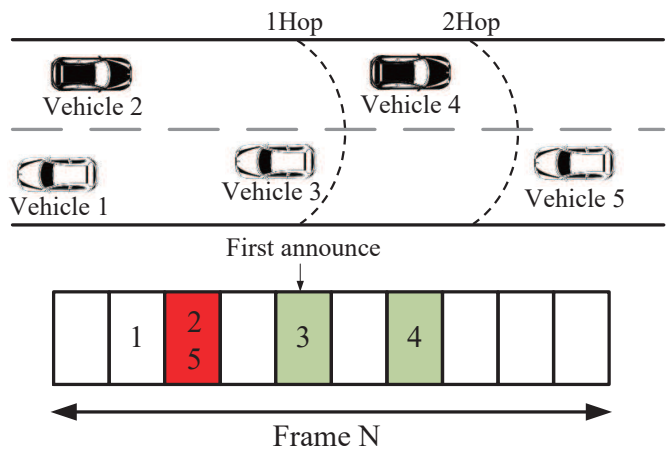

Figure 6. Collision prediction of prediction-based Time Division Multiple Access (TDMA) MAC (PTMAC).

In [11], MAC protocols designed or adapted for VANET were studied giving a qualitative comparison with the IEEE 802.11 protocols based on CSMA/CA and inter-frames spaces. The author of [12] proposed MOvement Prediction based Routing (MOPR) for VANETs, which improves the routing process by selecting the most stable route in terms of lifetime considering the movement of vehicles, and the MOPR was applied to position-based routing protocol. The results showed that the MOPR enhanced the performance compared to Greedy Perimeter Stateless Routing (GPSR) and MOvement-based Routing Algorithm (MORA). In [13,14], the authors suggested the hybrid protocol called Active Signaling-DTMAC (AS-DTMAC), with the purpose of resolving the problem of access collision in the time-slot assignments of the DTMAC protocol. In these works, AS-DTMAC is analyzed based on generating functions and the collision rate of AS-DTMAC is studied. The number of time frames to achieve collision-free resource allocation and the transmission requirements for urgent packets are studied. The simulations showed the enhanced performance of the proposed AS-DTMAC in terms of collision rate and convergence.

It is noted that VeMAC and CFR MAC can reduce resource collisions by using slot segmentation techniques. However, in the direction with high traffic density, the slot usage rate is low, resulting in frequent merging collisions. In addition, resource collisions between vehicles traveling in the same direction but at different speeds cannot be avoided. PTMAC suggests a resource collision method 
that is based on the prediction of location, speed, moving direction, and slot information of neighbor vehicles without using a slot partitioning method. However, many vehicles can not accurately predict whether or not there will be resource allocation collision (time-slot collision in TDMA-based VANET) due to sudden change of position arising from rapid acceleration/deceleration and moving direction change. In addition, in some road environments such as intersections with/without traffic lights, circuit intersections, and curved highways, the accuracy of the collision prediction can be greatly reduced if it relies only on the information of the current traveling speed and the simple moving direction (left and right or up and down) of each vehicle.

\section{Proposed Algorithm}

In this section, we propose a vector-based mobility model that can be used to predict resource collision in any kind of road environment, which considers speed, acceleration/deceleration, and the vector-based moving direction of each vehicle. In the proposed algorithm, each vehicle periodically broadcasts a FI message to its 1-hop neighbor vehicles through the control time-slot, like previous VeMAC, CFR MAC, and PTMAC. Neighbor vehicles receiving the FI message can predict the moving direction and the position of each vehicle through the proposed algorithm. In the following subsections, we explain the frame and FI message structures, a control time-slot occupation procedure, collision detection/prediction procedures, and a collision avoidance procedure, all of which are necessary to facilitate the proposed vector-based mobility model.

\subsection{Proposed Frame Structure and Fi Message Format}

Figure 7 shows the frame structure and FI message format to support the proposed algorithm. Each frame consists of $C$ control time-slots and $D$ data time-slots. Each vehicle searches for a control channel when entering the network and occupies one unused control time-slot. Each vehicle transmits an FI message through the occupied control channel. The FI message includes the ID of neighbor vehicles together with that of itself, the hop information of each vehicle, the traveling direction, the location (longitude and latitude), the occupied control time-slot, the current speed, and the acceleration. "ID Info" of each vehicle is used as the identification information. "Hop Info" expresses itself and the n-hop neighbor vehicle as " 0 " and " $\mathrm{n}$ ", respectively. "Direction Info" indicates how far the vehicle has rotated clockwise relative to the north at zero degrees. "Longitude and Latitude Info" are converted into $x, y$ coordinates using TM (Transverse Mercator) coordinates. "Time-slot Info" represents the occupied time-slot information of a vehicle. "Speed Info" is the speed of a vehicle, and "Acceleration Info" represents how much the speed of a vehicle has changed during the unit time.

\begin{tabular}{|c|c|c|c|c|c|c|c|c|c|c|}
\hline \multirow[b]{2}{*}{ Frame structure } & \multicolumn{5}{|c|}{ Control channel } & \multicolumn{5}{|c|}{ Data channel } \\
\hline & 1 & $\cdots$ & c & $\bullet$ & C & 1 & $\cdots$ & $d$ & $\bullet \bullet$ & D \\
\hline \multicolumn{11}{|c|}{ Example } \\
\hline \multirow{8}{*}{$\begin{array}{l}\text { Frame } \\
\text { Information } \\
\text { Message } \\
\text { structure }\end{array}$} & 2 & 5 & 10 & 25 & 9 & $\bullet \bullet \bullet$ & \multicolumn{2}{|c|}{ ID } & \multicolumn{2}{|l|}{ ID Info } \\
\hline & 0 & 1 & 1 & 2 & 3 & $\bullet \bullet$ & \multicolumn{2}{|c|}{ Hop } & \multicolumn{2}{|c|}{ Hop Info } \\
\hline & 0 & 1 & 1 & 1 & 1 & $\bullet \bullet$ & \multicolumn{2}{|c|}{ Direction } & \multirow{2}{*}{\multicolumn{2}{|c|}{$\begin{array}{l}\text { Direction Info } \\
\text { Latitude Info }\end{array}$}} \\
\hline & +343 & +382 & +394 & +374 & +384 & $\bullet \bullet$ & \multicolumn{2}{|c|}{ Latitude } & & \\
\hline & -372 & -392 & -283 & -384 & -385 & $\bullet \bullet$ & \multicolumn{2}{|c|}{ Longitude } & \multicolumn{2}{|c|}{ Longitude Info } \\
\hline & 3 & 2 & 1 & 65 & 3 & $\bullet \bullet$ & \multicolumn{2}{|c|}{ Time slot } & \multicolumn{2}{|c|}{ Time slot Info } \\
\hline & $52 \mathrm{~m} / \mathrm{s}$ & $0 \mathrm{~m} / \mathrm{s}$ & $18 \mathrm{~m} / \mathrm{s}$ & $7 \mathrm{~m} / \mathrm{s}$ & $4 \mathrm{~m} / \mathrm{s}$ & $\bullet \bullet$ & \multicolumn{2}{|c|}{ Speed } & \multicolumn{2}{|c|}{ Longitude Info } \\
\hline & $1 \mathrm{~m} / \mathrm{s}^{2}$ & $3 \mathrm{~m} / \mathrm{s}^{2}$ & $4 m / s^{2}$ & $2 \mathrm{~m} / \mathrm{s}^{2}$ & $0 \mathrm{~m} / \mathrm{s}^{2}$ & $\bullet \bullet$ & Accele & tion & \multicolumn{2}{|c|}{ Time slot Info } \\
\hline
\end{tabular}

Figure 7. Frame structure and frame information (FI) message format. 


\subsection{Control Time-Slot Occupation and Collision Recognition Procedures}

Each vehicle searches for the control channel at the initial network entry, analyzes the frame information message received in the control time-slot, and occupies the control time-slot to be used. At this time, the empty control time-slot are randomly selected by the vehicle to prevent collision of control time-slot allocation. If a control time-slot assignment is successfully made, this control time-slot assignment information is normally represented in the FI messages of neighbor vehicles. However, if collision of the control time-slot occurs, the neighbor vehicle does not update its control time-slot allocation information. In this way, each vehicle can judge whether there is a collision of its control time-slot allocation through the FI messages of neighbor vehicles. If a control time-slot allocation collision occurs, 2-hop neighbor vehicles attempt to re-occupy one of the unassigned control time-slots.

\subsection{Collision Prediction and Avoidance Procedures}

The vehicle that successfully occupies the control time-slot periodically broadcasts an FI message through the corresponding control time-slot every frame. Each vehicle updates the FI message received from the 1-hop neighbor vehicle together with its FI message and broadcasts it again. Suppose that there are five vehicles as shown in Figure 8. Vehicles 2 and 5 are 1-hop neighbors, vehicle 3 is a 2-hop neighbor, and vehicle 4 is a 3-hop neighbor of vehicle 1 . The third time-slot is reused by both vehicles 1 and 4, which are 3 hops away from each other. Vehicle 1 broadcasts the FI message including the information of its 1-hop neighbors, vehicles 2 and 5 . And then, vehicle 2 broadcasts its FI message including the information of vehicles 1,2 and 5, to vehicle 3 . Vehicle 3 broadcast its FI message to vehicle 4 , and vehicle 4 can recognize that the same time-slot is shared by its 3-hop neighbor, vehicle 1 . Then vehicle 4 changes its time-slot to another time-slot to avoid collision. In this manner, the information of a vehicle is spread over all the network, so the information of each vehicle in the network can be theoretically shared across the network. However, considering the latency required to relay FI messages and the network topology change that might occur during this latency, it is unrealistic and inefficient to share the information of each vehicle across all vehicles in a network. Therefore, when constructing the FI message, it is necessary to consider the information acquisition of the vehicle within the 3-hop communication range, in which the possibility of merging collision is high. Therefore, in this study, we limit the maximum value of "Hop info" in the FI message by 2, so that the neighbor vehicles updated by the FI messages are located equal to or less than two hops away, and consequently each vehicle receiving the FI message can recognize up to 3-hops neighbor vehicles.

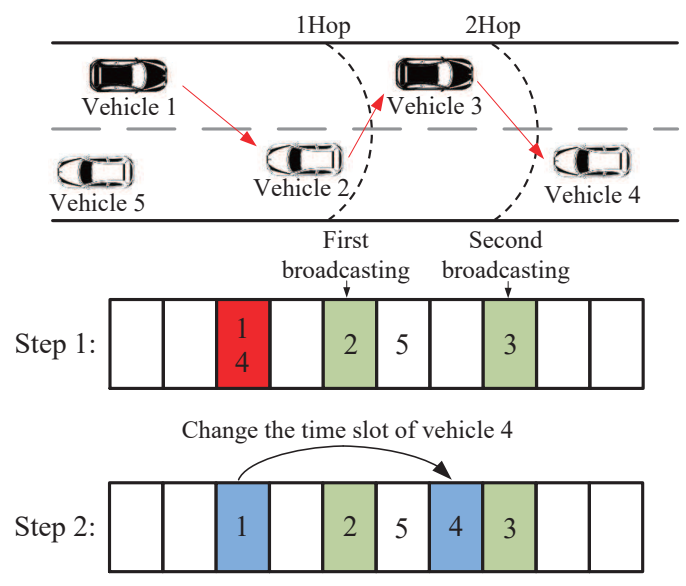

Figure 8. Broadcasting procedure.

The proposed resource collision prediction and merging collision avoidance procedures are as follows. Each vehicle verifies the existence of vehicles that use the same time-slot to that of itself. If a vehicle finds a 3-hop neighbor vehicle that uses the same time-slot as that of itself, the position of the vehicle at the next frame is periodically estimated using the proposed mobility model. When it is 
expected that the distance between two vehicles that share the same time-slot will be close to within a 2-hop distance, each vehicle gives up its own occupied time-slot and occupies a new time-slot to avoid a merging collision in the next frame.

\subsection{Mobility Model}

It is assumed that each vehicle knows its own information about vehicle speed, acceleration, and current position, which are obtained from a GPS device installed in the vehicle. The vehicle speed, acceleration, and current position at time $t$ are vectorized in $\mathrm{x}$ and y coordinates on the network, and denoted as $\vec{v}_{t}, \vec{a}_{t}$, and $\vec{r}_{t}$, respectively, as shown in Figure 9. Its moving position $\vec{r}_{t+\Delta t}$ after time $\Delta t$ through the vehicle information at time $t$ is calculated by

$$
\vec{r}_{t+\Delta t}=\vec{r}_{t}+\left(\vec{v}_{t}+\frac{1}{2} \vec{a}_{t} \Delta t^{2}\right) .
$$

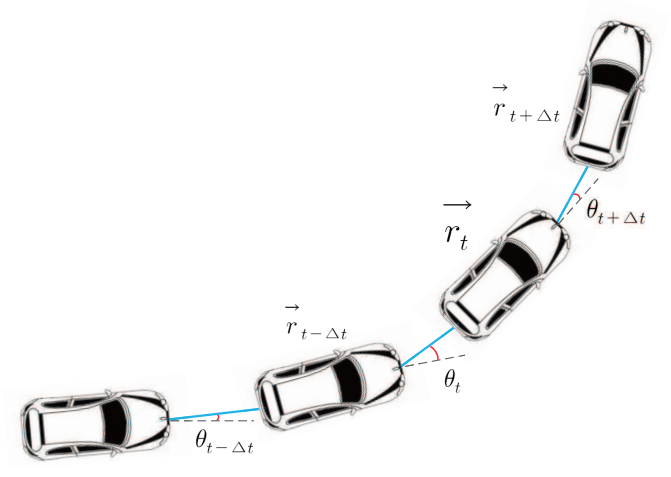

Figure 9. Mobility model.

The moving direction angle at time $t \theta(t)$ is estimated from previous change in the moving position measured from time $t-\Delta t$ to time $t$, which is given by

$$
\theta(t)=\arccos \frac{\vec{r}_{t} \cdot \vec{r}_{t-\Delta t}}{\left|\vec{r}_{t}\right| \cdot\left|\vec{r}_{t-\Delta t}\right|} .
$$

$\alpha_{t}$ is predicted through the change of $\theta(t)$ from time $t-\Delta t$ to time $t$ as

$$
\alpha_{t}=\theta(t)-\theta(t-\Delta t)
$$

$\tau_{t}$ is obtained through the change of $\alpha_{t}$ from time $t-\Delta t$ to time $t$ as

$$
\tau_{t}=\alpha_{t}-\alpha_{t-\Delta t}
$$

From (1)-(4), the expected direction $\theta(t+\Delta t)$ of the vehicle movement at time $t+\Delta t$ is given by

$$
\theta(t+\Delta t)=\theta(t)+\left(\alpha_{t}+\frac{1}{2} \tau_{t} \Delta t^{2}\right)
$$

The expected position of the vehicle at time $t+\Delta t$ is calculated by

$$
V_{t+\Delta t}=\vec{r}_{t+\Delta t} \cdot\left(\begin{array}{c}
\cos \theta(t+\Delta t) \\
\sin \theta(t+\Delta t)
\end{array}\right)
$$

\section{Performance Analysis}

In this section, we analyze the access/merging collision rates. The parameters used are summarized in Table 1. 
Table 1. Parameters.

\begin{tabular}{cl}
\hline Parameter & Definition \\
\hline$M_{m c}$ & The number of vehicles in a region where a merging collision may occur \\
$A_{a c}$ & The number of vehicles that generate a merging collision \\
$w$ & Road width \\
$l$ & Road length \\
$R$ & Transmission range \\
$d_{i j}(t)$ & The distance between vehicle $i$ and vehicle $j$ at time $t$ \\
$n_{i}(t)$ & Time slot number occupied by vehicle $i$ \\
$x(t)$ & x-axis coordinates of the vehicle \\
$y(t)$ & $y$-axis coordinates of the vehicle \\
$\theta_{i}(t)$ & The angle in the moving directions of the vehicles $i$ at time $t$ \\
$\theta_{i j}(t)$ & The angle difference in the moving directions of the vehicles $i$ and $j$ at time $t$ \\
$N_{e}$ & The number of empty time-slots in a frame \\
$N$ & Total number of time-slots in a frame \\
\hline
\end{tabular}

It is noted that the distance between vehicle $i$ and vehicle $j$ at time $t+\Delta t$ is estimated as

$$
\begin{aligned}
d_{i j}(t+\Delta t) & =\left[\left(x_{i}(t)+v_{i}(t) \Delta t \cos \theta_{i}(t)-x_{j}(t)-v_{j} \Delta t \cos \theta_{j}(t)\right)^{2}\right. \\
& \left.+\left(y_{i}(t)+v_{i}(t) \Delta t \sin \theta_{i}(t)-y_{j}(t)-v_{j} \Delta t \sin \theta_{j}(t)\right)^{2}\right]^{1 / 2}
\end{aligned}
$$

\subsection{Merging Collision Probability}

At any time $t$, the probability of a merging collision of vehicle $i$ is defined as $P_{M C}(i, t)$ and is calculated as

$$
p_{M C}(i, t)=M_{m c} \cdot \frac{2 R}{l} \cdot P_{m c}(i, j, t) .
$$

In Equation (7), $P_{m c}(i, j, t)$ represents the probability that vehicle $i$ will cause a merging collision with vehicle $j$ during time $\Delta t$, and is calculated as

$$
P_{m c}(i, j, t)=\operatorname{Pr}\left\{d_{i j}(t)>2 R\right\} \cdot \operatorname{Pr}\left\{d_{i j}(t+\Delta t) \leq 2 R\right\} \cdot \operatorname{Pr}\left\{n_{i}(t)=n_{j}(t)\right\} .
$$

The probability that the distance difference between vehicle $i$ and vehicle $j$ is larger than a 2-hop transmission range is calculated by

$$
\operatorname{Pr}\left\{d_{i j}(t)>2 R\right\}=1-\frac{2 R}{l} .
$$

Suppose that the distance between vehicles $i$ and $j$ is given by $d_{i j}(t)$ at time $t$. The maximum and minimum possible distances that vehicle $i$ can travel by during $\Delta t$ in a direction parallel to the road are estimated as $\Delta t \cdot v_{\max }$ and $\Delta t \cdot v_{\min } \cos \theta_{i}(t)$, respectively. The distance that vehicle $j$ travels by during $\Delta t$ in a direction parallel to the road is $\Delta t \cdot v_{j}(t) \cos \theta_{j}(t)$. To satisfy the condition of $d_{i j}(t+\Delta t) \leq 2 R, d_{i j}(t)+\Delta t \cdot v_{j}(t) \cos \theta_{j}(t)-2 R$ should be located between $\Delta t \cdot v_{\min } \cos \theta_{i}(t)$ and $\Delta t \cdot v_{\max }$. From Figure 10, the probability that the distance difference between two vehicles at time $t+\Delta t$ is less than a 2 -hops is calculated by

$$
\operatorname{Pr}\left\{d_{i j}(t+\Delta t) \leq 2 R\right\}=\frac{v_{\max }-v_{j}(t) \cos \theta_{j}(t)}{v_{\max }-v_{\min } \cos \theta_{i}(t)}+\frac{2 R-d_{i j}(t)}{\Delta t\left(v_{\max }-v_{\min } \cos \theta_{i}(t)\right)}
$$




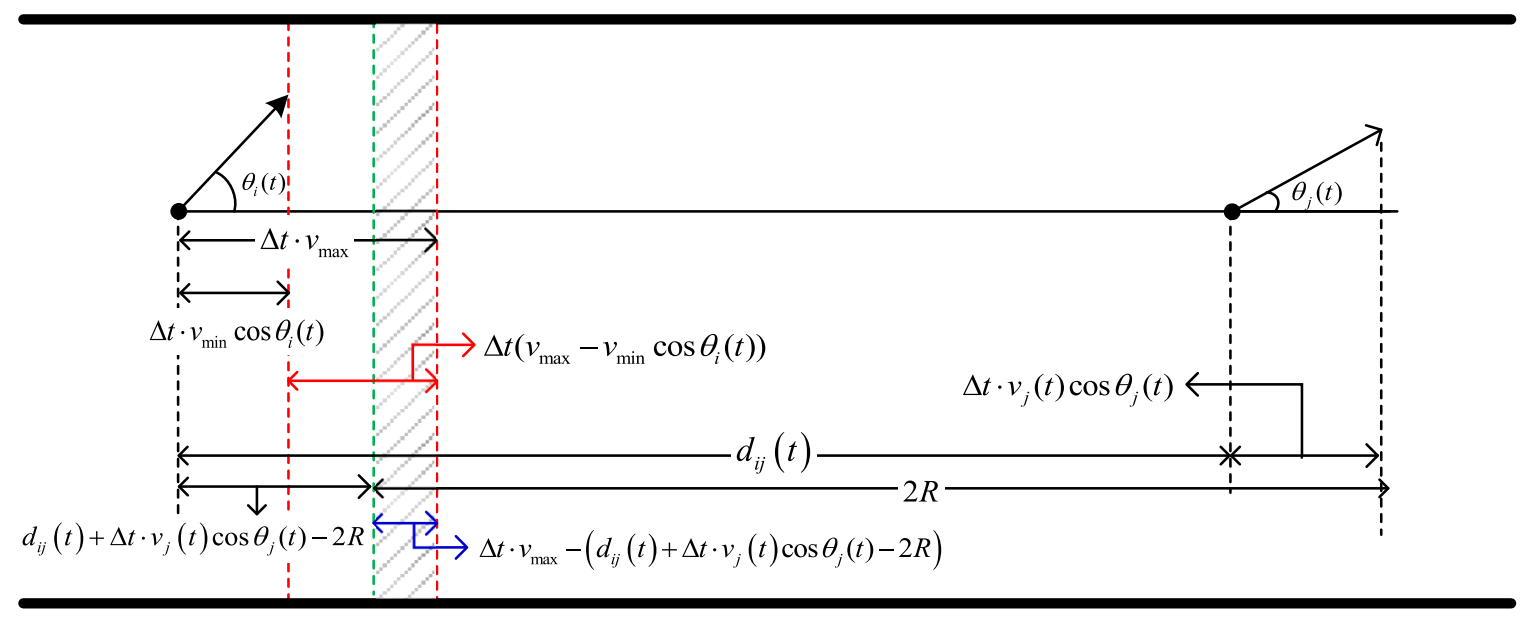

Figure 10. Illustration of the probability that the distance between vehicles $i$ and $j$ are shorter than $2 R$ at time $t+\Delta t$.

The probability that two vehicles occupy the same time-slot is given by

$$
\operatorname{Pr}\left\{n_{i}(t)=n_{j}(t)\right\}=\frac{1}{N}
$$

Substituting Equations (9)-(11) into Equation (8), the probability of merging collision is calculated by

$$
P_{m c}(i, j, t)=\left(1-\frac{2 R}{l}\right)\left(\frac{v_{\max }-\Delta t \cdot v_{j}(t) \cos \theta_{j}(t)}{v_{\max }-v_{\min } \cos \theta_{i}(t)}+\frac{2 R-d_{i j}(t)}{\Delta t\left(v_{\max }-v_{\min } \cos \theta_{i}(t)\right)}\right) \frac{1}{N}
$$

\subsection{Access Collision Probability}

The probability of an access collision of vehicle $i$ occurring at time $t$ is defined as $P_{A C}(i, t)$ and is calculated as

$$
P_{A C}(i, t)=A_{a c} \cdot \frac{2 R}{l w} \cdot P_{a c}(i, j, t) .
$$

The probability that vehicle $i$ will have an access collision due to vehicle $j$ at time $t$ is given by

$$
P_{a c}(i, j, t)=\left(1-\operatorname{Pr}\left\{N_{e}>A_{a c}\right\}\right) \cdot \operatorname{Pr}\left\{n_{i}(t)=n_{j}(t)\right\}
$$

The probability that the number of empty slots is larger than the number of vehicles attempting to occupy the new resource is given by

$$
\operatorname{Pr}\left\{N_{e}>A_{a c}\right\}=\left\{\begin{array}{ll}
\Delta t\left(\frac{1}{N_{e}}\right)^{A_{a c}-1} & \text { if } A_{a c}>1 \\
0 & \text { otherwise }
\end{array} .\right.
$$

The probability that vehicle $i$ and $j$ occupy the same time-slot becomes

$$
\operatorname{Pr}\left\{n_{i}(t)=n_{j}(t)\right\}=\frac{1}{N}
$$

Substituting (15) and (16) into (14), the probability of access collision is calculated by

$$
P_{a c}(i, j, t)=\frac{1}{N}\left(1-\Delta t\left(\frac{1}{N_{e}}\right)^{A_{a c}-1}\right) .
$$




\section{Simulation Results}

In this section, we compare the performance of the proposed algorithm with those of VeMAC, CFR MAC, and PTMAC. For the performance evaluation in diverse environments, we measure the mobility of a vehicle using GPS equipment called VBOX Sport.

Figure 11 shows VBOX Sport and its management program. VBOX Sport records the actual vehicle path, speed, and location every $50 \mathrm{~ms}$ via GPS and is used to derive the actual mobility model of the vehicle in this paper. In order to collect measurement data in various environments, we collected data repeatedly from actual highways and intersections, taking into account dozens of moving cases such as acceleration/deceleration, stopping, high speed and low speed. Figures 12 and 13 show road scenarios for an intersection with traffic lights and a circuit intersection without traffic lights, respectively. It is assumed that the signal of the traffic lights on the road changes every $10 \mathrm{~s}$. At an intersection, the vehicle's direction of travel can be a left turn, a right turn, or moving forward, where the probability that the vehicle will choose any direction of travel is the same as $1 / 3$. At a circuit intersection, it is assumed that each vehicle can proceed in one of four directions of 90, 180, 270, and 360 degrees, with the same probability of $1 / 4$ as well. Table 2 summarizes the remaining simulation parameters used in this paper.



Figure 11. VBOX Sport GPS device and management program.

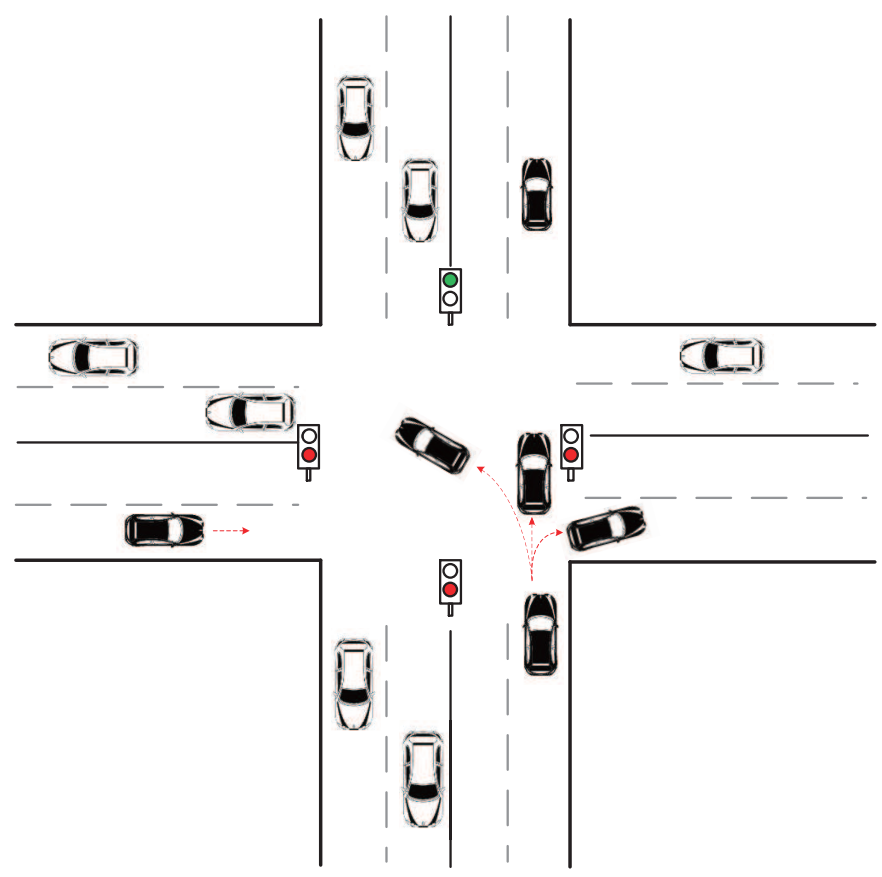

Figure 12. Intersection with traffic lights. 


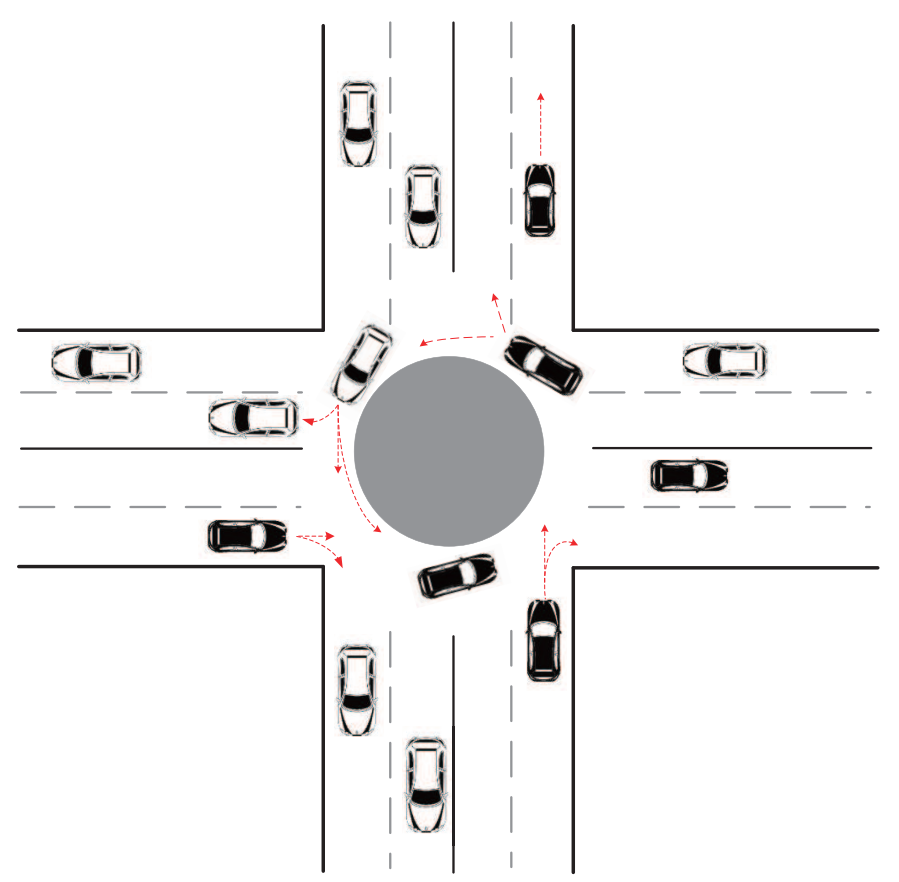

Figure 13. Circuit intersection without traffic lights.

Table 2. Simulation parameter.

\begin{tabular}{ccc}
\hline Parameter & Highway & Intersection \\
\hline Vehicles speed & $13.8 \sim 41.6 \mathrm{~m} / \mathrm{s}$ & $0 \sim 41.6 \mathrm{~m} / \mathrm{s}$ \\
Road width & $80 \mathrm{~m}$ & $80 \mathrm{~m}$ \\
Road length & $2000 \mathrm{~m}$ & $1000 \mathrm{~m}$ \\
The number of vehicles & $80 \sim 300(\mathrm{step}=20)$ & $80 \sim 300(\mathrm{step}=20)$ \\
Traffic light & - & $10 \mathrm{~s}$ \\
Frame size & $100 \mathrm{~ms}$ & $100 \mathrm{~ms}$ \\
The number of control time-slot & 100 & 100 \\
Time-slot duration & $1 \mathrm{~ms}$ & $1 \mathrm{~ms}$ \\
Time rate of change & $0.1 \mathrm{~s}$ & $0.1 \mathrm{~s}$ \\
Transmission range & $150 \mathrm{~m}$ & $150 \mathrm{~m}$ \\
\hline
\end{tabular}

Regarding the performance metrics, we evaluate merging collision rate and access collision rate as a function of the number of vehicles in a network. Merging and access collision rates are defined as the average number of merging collisions per frame and the average number of access collisions per frame, respectively.

Figures 14-16 show the results for the average merging collision rate as a function of the number of vehicles in various environments. Figure 14 shows the merging collision rate on the highway. The result shows that the proposed algorithm decreases merging collision rate by up to $55.1 \%, 40.8 \%$ and $11.7 \%$ compared to VeMAC, CFR MAC, and PTMAC, respectively, which shows that the vector-based prediction model outperforms slot partitioning-based algorithms. Because PTMAC and the proposed algorithm do not use the slot partitioning method, merging collisions can occur between vehicles moving in different directions. However, the result also verifies that the proposed algorithm prevents merging collision more effectively than PTMAC. Figure 15 shows the merging collision rate at an intersection with traffic lights. In this scenario, the proposed algorithm reduces the merging collision rate by up to $63.3 \%, 46.9 \%$, and $25.2 \%$, compared to VeMAC, CFR MAC, and PTMAC, respectively. When the traffic light is red, there are many vehicles stopping, resulting in fewer merging collisions. On the other hand, when the traffic light is green and many vehicles pass through the intersection, there is a high possibility that resource collision will occur due to high mobility and the various moving directions of vehicles. As a result, the merging collision rate increases in VeMAC and CFR MAC using 
the slot partitioning method. On the other hand, in PTMAC, the merging collision rate increases because each vehicle cannot accurately predict the moving direction of neighbor vehicles. Figure 16 shows the merging collision rate at a circuit intersection. In this scenario, the proposed algorithm reduces the merging collision rate by up to $49 \%, 25.8 \%$, and $14.9 \%$, compared with VeMAC, CFR MAC, and PTMAC, respectively, and this result can be explained by the same reason as for the explanation of Figure 15. In addition, the proposed algorithm decreases the merging collision rate compared to VeMAC, CFR MAC, and PTMAC, and this performance gain is the largest for the highway and the smallest for the circuit intersection without traffic lights. To sum up, the proposed algorithm has a greater effect in decreasing merging collision rates when the traffic density is high and the mobility of the vehicle is high with rapidly changing travel direction.

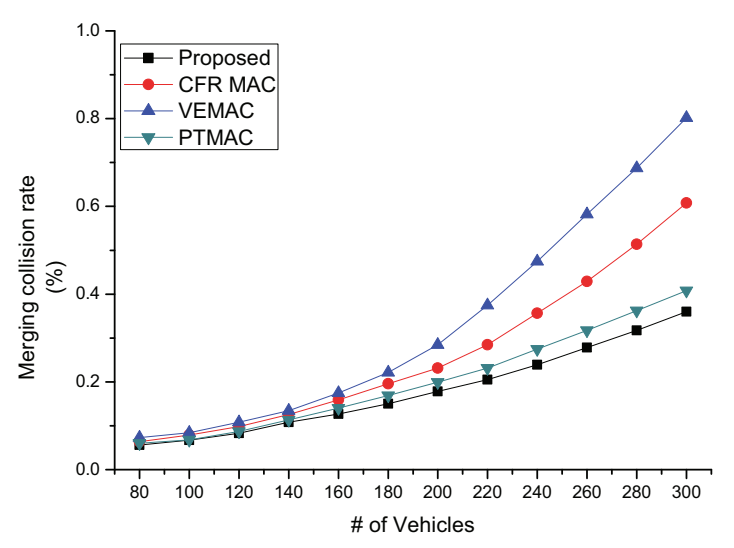

Figure 14. Merging collision rate for a highway.

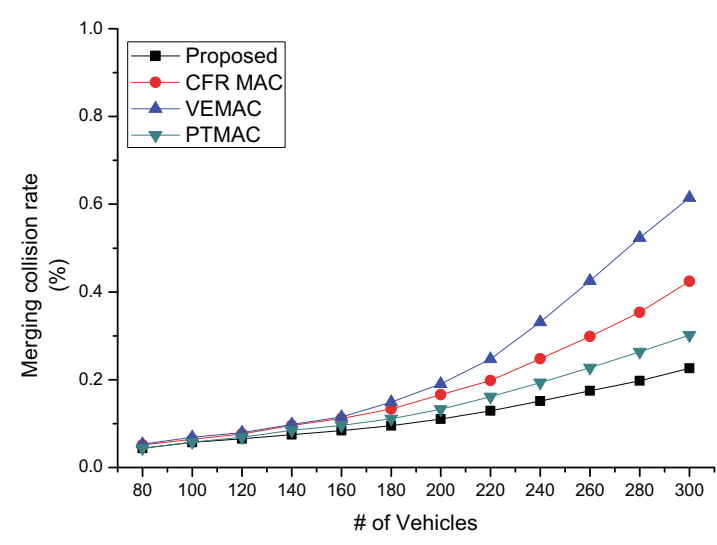

Figure 15. Merging collision rate for an intersection with traffic lights. 


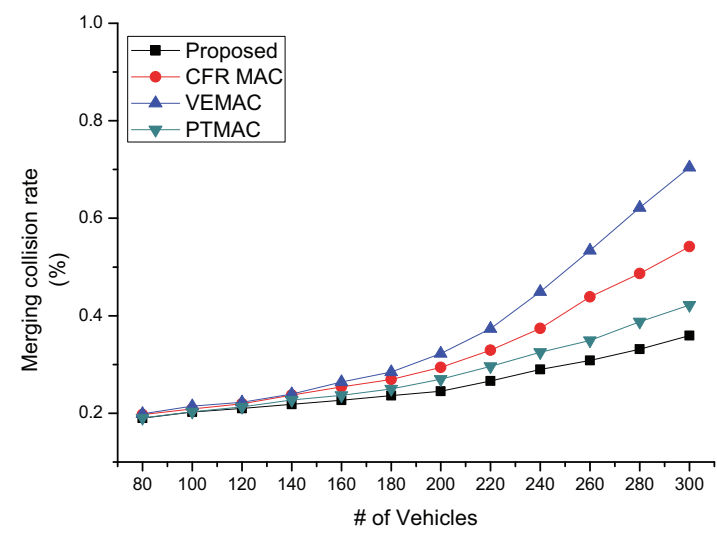

Figure 16. Merging collision rate for a circuit intersection without traffic lights.

Figures 17-19 show the results for the average access collision rates as a number of vehicles in various environments. In Figure 17, it can be seen that the proposed algorithm in the highway environment reduces the access collision by up to $60.5 \%, 44.6 \%$, and $17.9 \%$, compared to VeMAC, CFR MAC, and PTMAC, respectively. In Figure 18, we can see that the proposed algorithm in the intersection with traffic lights reduces access collisions by up to $60.8 \%, 44.6 \%$, and $23.4 \%$, compared with VeMAC, CFR MAC, and PTMAC, respectively. In Figure 19, the result shows that the proposed algorithm in the circuit intersection without traffic lights reduces access collisions by up to $61.1 \%, 45 \%$ and $23.9 \%$, compared with VeMAC, CFR MAC, and PTMAC, respectively. It is noticed that access collision is incurred by vehicles that simultaneously enter the network and occupy the time-slot, or by vehicles that experience merging collision and try to re-occupy a time-slot. Similar to the results of the merging collision rate, the proposed algorithm decreases access collision rate compared to VeMAC, CFR MAC, and PTMAC and this performance gain is largest for the highway and smallest for the circuit intersection without traffic lights. As such, the same conclusion can be made as in the case of the merging collision: the proposed algorithm has a greater effect on decreasing the access collision rate as the traffic density is high and the mobility of vehicles is high with rapidly changing travel direction.

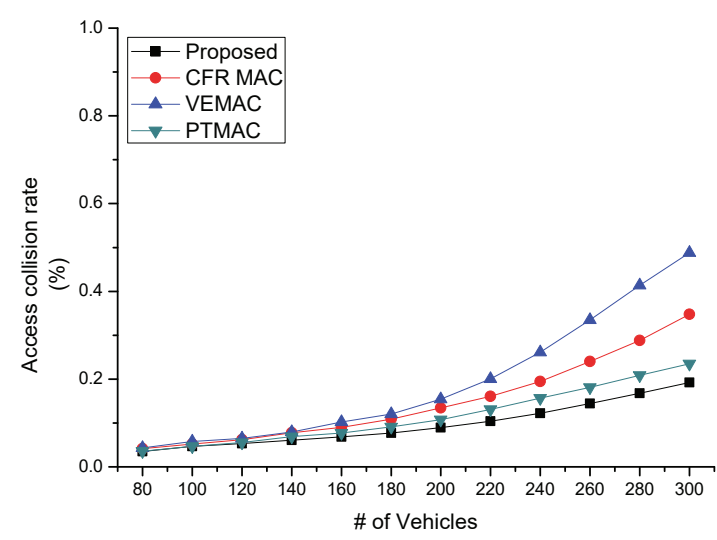

Figure 17. Access collision rate on a highway. 


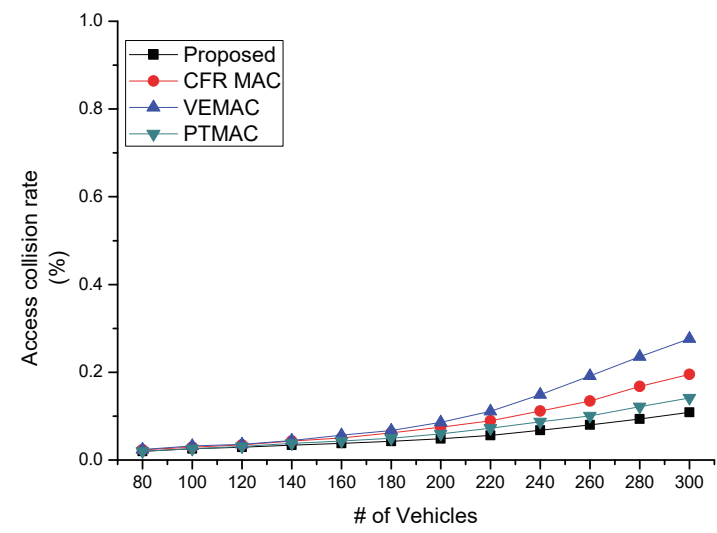

Figure 18. Access collision rate of an intersection with traffic lights.

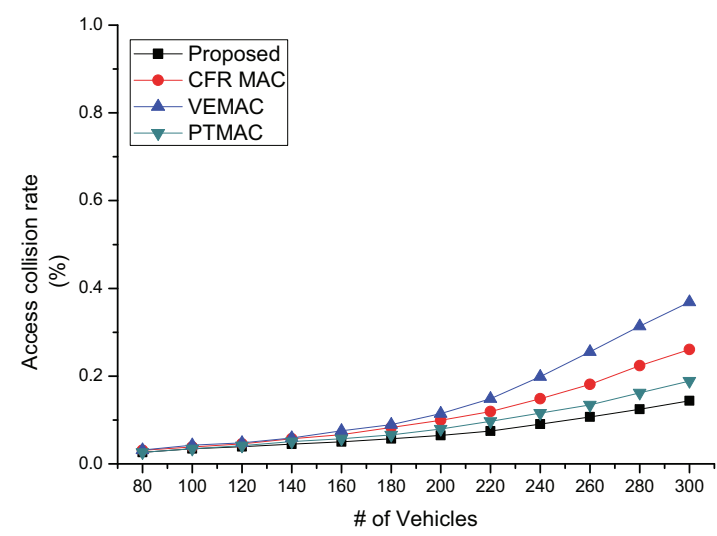

Figure 19. Access collision rate of a circuit intersection without traffic lights.

Figures 20 and 21 show both the simulation and analysis results for the merging and access collision rates of the proposed algorithm in various environments. In these figures, we can verify that the analysis results coincide with the simulation results. Figure 20 shows that the differences between analysis and simulation results for merging collision rates are at most $1.24 \%, 0.79 \%$, and $0.51 \%$ for a highway, an intersection with traffic lights, and a circuit intersection without traffic lights, respectively. From Figure 21, we can see that the differences between analysis and simulation results for merging collision rates are at most $0.87 \%, 0.64 \%$, and $0.59 \%$ for a highway, an intersection with traffic lights, and a circuit intersection without traffic lights, respectively.

From the results shown in Figures 14-19, we can verify the performance enhancement of the proposed algorithm over other protocols. It is noticed that the proposed algorithm is a prediction-based algorithm and the performance improvement of the proposed algorithm against PTMAC (another prediction-based algorithm) is greater under the circuit or intersection than under the two-way freeway road, which shows the effectiveness of the proposed algorithm on roads with great freedom of movement. 


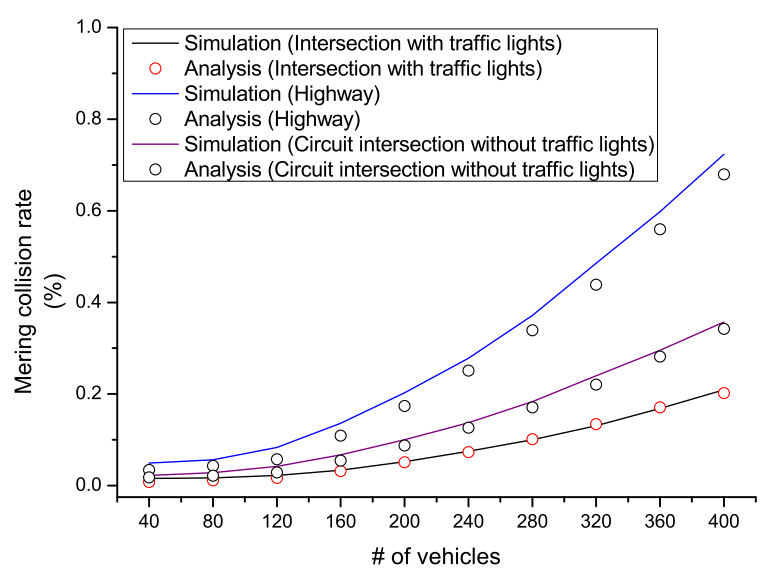

Figure 20. Rate of merging collision under the proposed algorithm.

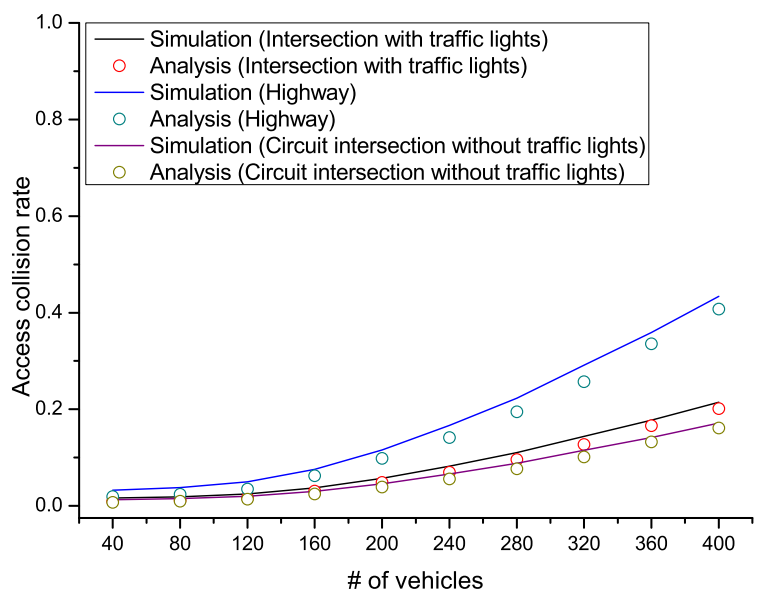

Figure 21. Rate of access collision under the proposed algorithm.

\section{Conclusions}

In this paper, we propose a vector-based mobility prediction model that aims to avoid merging or access collisions between vehicles by predicting the anticipated movement position and direction of each vehicle. The frame and the FI message structures to support the proposed algorithm are proposed and the procedures for control time-slot occupation, collision prediction/avoidance are explained. The performance of the proposed algorithm is evaluated in various road environments such as a highway, an intersection with/without traffic lights, and a circuit intersection. The results show that the proposed algorithm reduces access and merging collisions compared to existing PTMAC, VeMAC, and CFR MAC algorithms. In particular, it has been shown that the performance gain of the proposed algorithm is enhanced in road environments where the traffic density is high and where vehicles have high mobility and change their travel directions frequently.

Author Contributions: J.-H.B. contributed to the paper by deriving the results of the simulation and by making the draft version of the paper. J.-R.L. was responsible for the main idea, theoretical analysis, coordination and proof reading of the paper. All authors have read and agreed to the published version of the manuscript.

Acknowledgments: This research was supported by the MSIT(Ministry of Science and ICT), Korea, under the ITRC(Information Technology Research Center) support program (IITP-2020-2018-0-01799) supervised by the IITP (Institute for Information \& communications Technology Planning \& Evaluation), and by the National Research Foundation of Korea(NRF) grant funded by the Korea government(MEST) (No.NRF-2020R1A2C1010929).

Conflicts of Interest: The authors declare no conflict of interest. 


\section{Reference}

1. Motor Vehicle Collision Annual Report. Edmonton. 2016. Available online: https://www.edmonton.ca/ transportation/RoadsTraffic/2016MVCAnnualReportsm.pdf (accessed on 18 June 2020)

2. Kumar, R.; Dave, M. Mobile Agent as an Approach to Improve QoS in Vehicular Ad Hoc Network. arXiv 2011, arXiv:1108.2095.

3. Hadded, M.; Muhlethaler, P.; Laouiti, A.; Zagrouba, R. TDMA-Based MAC Protocols for Vehicular Ad Hoc Networks: A Survey, Qualitative Analysis, and Open Research Issues. IEEE Commun. Surv. Tutor. 2015, 17, 2461-2492, doi:10.1109/COMST.2015.2440374. [CrossRef]

4. Zhuo, X.; Hua, S.; Miao, L.; Dai, Y. Direction matters: A decentralized direction-based tdma scheduling strategy for VANET. In Proceedings of the 2011 IEEE 13th International Conference on Communication Technology, Jinan, China, 25-28 September 2011; pp. 566-571, doi:10.1109/ICCT.2011.6157940. [CrossRef]

5. Zhang, R.; Cheng, X.; Yang, L.; Shen, X.; Jiao, B. A Novel Centralized TDMA-Based Scheduling Protocol for Vehicular Networks. IEEE Trans. Intell. Transp. Syst. 2015, 16, 411-416, doi:10.1109/TITS.2014.2335746. [CrossRef]

6. Almalag, S.; Olariu, S.; Weigle, C. TDMA cluster-based mac for VANETs (TC-MAC). In Proceedings of the 2012 IEEE International Symposium on a World of Wireless, Mobile and Multimedia Networks (WoWMoM), San Francisco, CA, USA, 25-28 June 2012; pp. 1-6, doi:10.1109/WoWMoM.2012.6263796. [CrossRef]

7. Borgonovo, F.; Capone, A.; Cesana, M.; Fratta, L. ADHOC MAC: New MAC architecture for ad hoc networks providing efficient and reliable point-to-point and broadcast services. Wirel. Netw. 2004, 10, 359-366, doi:10.1109/WCNC.2003.1200502. [CrossRef]

8. Omar, H.A.; Zhuang, W.; Li, L. VeMAC: A TDMA-Based MAC Protocol for Reliable Broadcast in VANETs. IEEE Trans. Mob. Comput. 2013, 12, 1724-1736, doi:10.1109/TMC.2012.142. [CrossRef]

9. Zou, R.; Liu, Z.; Zhang, L.; Kamil, M. A near collision free reservation based MAC protocol for VANETs. In Proceedings of the 2014 IEEE Wireless Communications and Networking Conference (WCNC), Istanbul, Turkey, 6-9 April 2014; pp. 1538-1543, doi:10.1109/WCNC.2014.6952438. [CrossRef]

10. Jiang, X.; Du, D. PTMAC: A Prediction-Based TDMA MAC Protocol for Reducing Packet Collisions in VANET. IEEE Trans. Veh. Technol. 2016, 65, 9209-9223.

[CrossRef]

11. Menouar, H.; Filali, F.; Lenardi, M. A survey and qualitative analysis of mac protocols for vehicular ad hoc networks. IEEE Wirel. Commun. 2006, 13, 30-35.

[CrossRef]

12. Menouar, H.; Lenardi, M.; Filali, F. Movement prediction-based routing (MOPR) concept for position-based routing in vehicular networks. In Proceedings of the IEEE 66th Vehicular Technology Conference, Baltimore, MD, USA, 30 September-3 October 2007.

13. Boukhalfa, F.; Hadded, M.; Muhlethaler, P.; Shagdar, O. An active signaling mechanism to reduce access collisions in a distributed TDMA based MAC protocol for vehicular networks. In Proceedings of the Advanced Information Networking and Applications (AINA-2019), Kunibiki Messe, Japan, 27-29 March 2019.

14. Boukhalfa, F.; Hadded, M.; Muhlethaler, P.; Shagdar, O. An Analytical Model for Performance Analysis of an Active Signaling-based TDMA MAC Protocol for Vehicular Networks. In Proceedings of the 2019 IEEE 90th Vehicular Technology Conference (VTC2019-Fall), Honolulu, HI, USA, 22-25 Sepember 2019.

(C) 2020 by the authors. Licensee MDPI, Basel, Switzerland. This article is an open access article distributed under the terms and conditions of the Creative Commons Attribution (CC BY) license (http://creativecommons.org/licenses/by/4.0/). 